\title{
Fish oils protects against cecal ligation and puncture-induced septic acute kidney injury via the regulation of inflammation, oxidative stress and apoptosis
}

\author{
ZHAOHENG LIN, JING JIN and XIYUN SHAN \\ Intensive Care Unit, The People's Hospital of Xishuangbanna Dai Nationality Autonomous Prefecture, \\ Jinghong, Yunnan 666100, P.R. China
}

Received April 30, 2019; Accepted August 14, 2019

DOI: $10.3892 /$ ijmm.2019.4337

\begin{abstract}
Septic acute kidney injury (AKI) is usually caused by sepsis. $\omega 3$ fatty acid has been reported to suppress sepsis-induced organ dysfunction to a certain degree. The present study aimed to investigate the effects of $\omega 3$ fatty acid in septic renal injury. Sprague Dawley rats were used to establish a cecal ligation and puncture (CLP) model in order to mimic the development of septic injury. The rats were treated with dexamethasone and fish oils (FOs) for 4 days prior to CLP. Alterations in the morphology of the tissues, the renal function and the induction of inflammation, oxidative stress and apoptosis were evaluated. The effects of FOs on nuclear factor $-\kappa \mathrm{B}(\mathrm{NF}-\kappa \mathrm{B}), \mathrm{JAK} 2 / \mathrm{STAT} 3$ and p38-MAPK were determined. The rats of the CLP model group exhibited low survival rates and increased expression of serum creatine, blood urea nitrogen, neutrophil gelatinase-associated lipocalin, kidney injury molecule-1 and of proinflammatory cytokines. In addition, the levels of the markers of oxidative injury and apoptosis were increased. The induction of renal injury was notably reversed by administration of dexamethasone and FOs. The expression levels of the protein markers involved in inflammation and apoptosis were measured and the results indicated that FOs inhibited JAK/STAT3 and p-38MAPK signaling, while they concomitantly increased the expression of NF- $\mathrm{kB}$. The present study highlighted that FOs improve CLP-induced mortality and renal injury by inhibiting inflammation, oxidative stress and apoptosis.
\end{abstract}

Correspondence to: Dr Zhaoheng Lin, Intensive Care Unit, The People's Hospital of Xishuangbanna Dai Nationality Autonomous Prefecture, 4 Galan South Road, Jinghong, Yunnan 666100, P.R. China

E-mail: linzhaoheng_dz@163.com

Key words: fish oils, septic renal injury, inflammation, oxidative stress, apoptosis

\section{Introduction}

Sepsis is a life-threatening organ dysfunction, caused by an overwhelming immune response to infection (1). The kidneys are frequently affected by sepsis, which results in the development of acute kidney injury (AKI), inducing unfavorable health outcomes. Previous epidemiological investigations have revealed that patients with AKI (mild or short) tend to have a higher risk of developing chronic- and end-stage kidney disease at later stages of their life (2). Volume resuscitation, antimicrobial therapy and renal replacement therapy remain the main types of treatment for sepsis-induced AKI (3). Recent investigations have focused on the supportive measures that aim to keep the patient alive.

$\omega 3$ fatty acids (FAs), eicosapentaenoic acid (EPA) and docosahexaenoic acid (DHA), are the main component of fish oils (FOs) (4). Previous studies have shown that enteral nutrition based on FOs affects the induction of sepsis, which is the leading cause of AKI $(5,6)$. Sepsis is a systemic inflammatory response and a severe postoperative infection, which is usually initiated by bacteria and toxins (7). Following the release of bacterial toxins, the immune response is activated, and organ disorders may develop (8). The sepsis-induced kidney inflammatory response involves the increased production of pro-inflammatory factors, which may contribute to high mortality (9). Concomitantly, the release of TNF $\alpha$, IL-1 $\beta$ and IL-6 contributes to the inflammatory factors (9). During the development of sepsis, the levels of these pro-inflammatory factors are significantly elevated at the early stage of disease onset affecting the induction of pathophysiological responses (10). $\omega-3$ FAs have shown considerable potential in the prevention of chronic kidney disease in humans (11). The $\omega-3$ fatty acids, especially EPA and DHA, exert anti-inflammatory effects in humans (12). Shih et al (13) demonstrated that FOs could suppress AKI and the inflammatory response in septic mice. The pathophysiology of sepsis remains controversial. In addition to inflammatory reactions, sepsis-induced AKI often involves the induction of oxidative stress and apoptosis $(14,15)$. Previously, the administration of an $\omega 3$-FA-rich infusion during parenteral nutrition was shown to alleviate sepsis in patients (16); however, the detailed mechanisms of this process remain unknown. 
Blood urea nitrogen (BUN) and serum creatine ( $\mathrm{SCr}$ ) are classic biomarkers for renal injury and have been reported as biomarkers for delayed kidney injury (17). Accompanied with increased $\mathrm{SCr}$ levels, the glomerular filtration rate (GFR) is reduced (18). The secretion of SCr contributes to $10-40 \%$ of total SCr; thus, reductions in GFR may be linked. Additionally, the concentrations of Scr are notably influenced by variable factors, including age, gender, diet and drugs (19). Thus, the levels of BUN and Scr are not sufficient for the diagnosis of early kidney injury. Kidney injury molecule-1 (KIM-1) and neutrophil gelatinase-associated lipocalin (NGAL) have been detected in early renal tubular injury (20). The expression of NGAL in both blood and urine have been reported to indicate the presence, severity and development of renal disease, especially in chronic renal disease (21). KIM-1 is a transmembrane protein not found in normal kidney; the expression of KIM-1 and NGAL has been linked to renal ischemia, which usually leads to AKI (22).

In the present study, the cecal ligation and puncture (CLP) model was established to induce sepsis. This model mimics the condition noted in patients with bowel perforation and polymicrobial infection (23). The effects of FOs on sepsis-induced inflammation, apoptosis and oxidative stress were investigated.

\section{Materials and methods}

Animals. Male Sprague Dawley rats ( $\mathrm{n}=32 ; 7$-weeks-old, Qinglongshan Experimental Animal Center, China) weighing 250-330 g were employed. A total of 3 rats were housed per cage and maintained in a 12 -h light/dark cycle at $25^{\circ} \mathrm{C}$. The experimental protocol was approved by the commission for animal experimentation of the People's Hospital of the Xishuangbanna Dai Nationality Autonomous Prefecture.

Following acclimation for 1 week, the rats with an initial body weight of $280 \pm 25 \mathrm{~g}$ were randomly assigned to one of the following groups: Sham-operated $(n=8)$ and CLP $(n=24)$. The former group was used as a control group and the latter as the experimental sepsis group. The CLP model group was randomly assigned to one of the following subgroups: CLP sepsis $(n=8)$ used for model animals, CLP treated with dexamethasone $[1 \mathrm{mg} / \mathrm{kg}$, intraperitoneal (i.p.) daily; $\mathrm{n}=8]$ used for positive control and CLP treated with FO-containing fat emul$\operatorname{sion}(2 \mathrm{ml} / \mathrm{kg}$ i.p. daily; $\mathrm{n}=8)$. The rat model was established by CLP-induced sepsis and intraperitoneally administered with dexamethasone or FOs (Table I) once a day. The treatment was provided 3 days prior to CLP operation and was continued until animal sacrifice. The serum samples were collected from the inner canthal orbital vein $24 \mathrm{~h}$ following CLP operation. All rats were sacrificed by using pentobarbital sodium ( $200 \mathrm{mg} / \mathrm{kg}$; i.p. injection) following $96 \mathrm{~h}$ of CLP operation. The collected renal tissues were harvested and all renal tissues from each rat was half embedded in paraffin and half stored in $-80^{\circ} \mathrm{C}$ refrigerator. Some animals did not survive the operation.

Sepsis renal injury was induced by CLP as previously described (24). Briefly, rats were fast with access to water for $12 \mathrm{~h}$ before the experiments. All rats were anesthetized with isoflurane inhalation. The hypogastric region was shaved and disinfected with alcohol, cutting $\sim 2 \mathrm{~cm}$ long in middle of abdomen. The cecum was subsequently exposed and ligated just below the ileocecal valve with a 3-0 silk and punctured with an 18-gauge needle. A droplet of feces was then squeezed from the puncture hole. The bowel was placed back into the abdominal cavity and the incision was sutured with 4-0 silk. The sham-operated rats underwent CLP surgery except cecal ligation and perforation. All rats were given $0.9 \%$ saline solution immediately after surgery for fluid resuscitation.

Survival rate assessment. In each group, the survival rate of the rats $(n=8)$ was evaluated over a period of 4 days following operation. Following CLP surgery, the rats were observed every $12 \mathrm{~h}$ for a total of $96 \mathrm{~h}$. The mortality percentage was recorded. All animals that did not survive the operation during the 12-24 h period was recorded. The human endpoint in this assay was determined according to previous investigation (25). All animals were monitored three times daily for humane endpoints and were euthanized when the humane endpoints were reached. Humane endpoints included loss of $20 \%$ weight from surgery, or the clinical score reached 5 . The scores were denoted based on hunched posture (0-2), ruffled coat $(0-2)$, diarrhea $(0-2)$, dehydrated eyes $(0-2)$, decreased body temperature (0-1) and reluctance to move (0-2).

Hematoxylin and eosin staining $(H \& E)$ staining. The induction of sepsis was performed via CLP and the rats were sacrificed following $96 \mathrm{~h}$ of treatment. Renal and small intestinal tissues were fixed in $4 \%$ formaldehyde at room temperature for $>24 \mathrm{~h}$ for histopathological analysis. Subsequently, the fixed tissues were embedded in paraffin. Sections $(5 \mu \mathrm{m})$ were sliced. After deparaffinization and rehydration, the sections were stained with hematoxylin (Sigma-Aldrich; Merck KGaA) for $5 \mathrm{~min}$ and then stained with eosin (Sigma-Aldrich) for $3 \mathrm{~min}$ at room temperature. The samples were subsequently visualized by optical microscopy (Olympus Corporation) at x100 magnification.

Assessment of serum and renal biochemical parameters. Blood biochemical analysis was performed according to previous investigations. The blood samples were extracted $24 \mathrm{~h}$ following sham or CLP operation and centrifuged at $1400 \mathrm{x}$ g to prepare serum at $4^{\circ} \mathrm{C}$ for $10 \mathrm{~min}$. $\mathrm{SCr}$ levels were evaluated by a creatinine enzymatic assay kit (MAK080-1KT, Sigma-Aldrich, Merck KGaA) and measured at $570 \mathrm{~nm}$. BUN was detected with an enzymatic assay kit (MAK006-1KT, Sigma-Aldrich, Merck KGaA) and measured at $570 \mathrm{~nm}$. The expression levels of NGAL (ab119602, Abcam) and KIM-1(ab119597, Abcam) were measured using corresponding kits and measured at $450 \mathrm{~nm}$. All measurements were conducted with a multiscan spectrum spectrophotometer (Thermo Fisher Scientific Inc.)

\section{Evaluation of inflammatory factors}

ELISA assays. The serum samples were extracted $24 \mathrm{~h}$ following sham or CLP operation. The expression levels of tumor necrosis factor (TNF)- $\alpha$ (ER006-96), interleukin (IL)-1 $\beta$ (ER008-96), and IL-6 (ER003-96) in the serum were determined by ELISA kits (Shanghai ExCell Biology, Inc.) following the manufacturer's protocol. The samples were centrifuged at $1,500 \mathrm{x}$ g for $15 \mathrm{~min}$ at $4^{\circ} \mathrm{C}$. Subsequently, the supernatant was collected, and the measurements were conducted at $450 \mathrm{~nm}$ using a multiscan spectrum spectrophotometer (Thermo Fisher Scientific Inc.). 
Immunofluorescence staining. The expression levels of TNF- $\alpha$, IL-1 $\beta$, and IL-6 were detected in renal and intestinal tissues by immunofluorescence. Sections $(5 \mu \mathrm{m})$ of renal and intestinal tissues were obtained. The samples were incubated with blocking solution (5\% bovine serum albumin in PBS) and permeabilized $(0.1 \%$ Triton $\mathrm{X}-100$ in PBS). Primary antibodies against TNF- $\alpha$ (1:100, ab6671, Abcam), IL-1 $\beta$ (1:100, ab9722, Abcam), and IL-6 (1:200, YB-0782R, Yubo Biological Technology Ltd.) were used for sequential double immunofluorescence staining for $2 \mathrm{~h}$ at room temperature in the dark. The goat anti-rabbit IgG H\&L (Alexa Fluor ${ }^{\circledR} 488$ ) secondary antibody (1:500, ab1500, Abcam) were conjugated with fluorescein isothiocyanate. The sections were mounted in an anti-fading agent (DAPI; Invitrogen; Thermo Fisher Scientific, Inc.) for $5 \mathrm{~min}$ at room temperature, and subsequently imaged and analyzed with a fluorescence microscope (Olympus Corporation) at x100 magnification.

Assessment of antioxidant activity and oxidative stress markers in renal tissues. The levels of oxidative stress biomarkers were determined according to a previous investigation (26). The renal tissues were homogenized in $50 \mathrm{mmol} / \mathrm{l}$ phosphate buffer, and subsequently centrifuged at $12,000 \mathrm{x}$ g for $20 \mathrm{~min}$ at $4^{\circ} \mathrm{C}$. The supernatant was collected from the samples for the detection of the markers of oxidative stress. The concentration of malondialdehyde (MDA) was detected with an MDA assay kit (Beijing Solarbio Life Sciences). The activity level of superoxide dismutase (SOD) and glutathione peroxidase (GSH-Px) were evaluated by commercial kits (Jiancheng Bioengineering Institute). All detection was conducted according to manufacturer's protocols.

Terminal deoxynucleotidyl-transferase-mediated dUTP nick end labelling (TUNEL) staining. The TUNEL assay was performed as described previously (27). The paraffin-embedded renal tissues were sliced into $5 \mu \mathrm{m}$ sections at room temperature. After deparaffinization, the samples were rehydrated with gradient concentration $(90,80,70 \%)$ of ethanol and immersed in $4 \%$ formaldehyde in PBS for $9 \mathrm{~min}$. Subsequently, the sections were treated with proteinase $\mathrm{K}(20 \mu \mathrm{g} / \mathrm{ml})$ and incubated in a nucleotide mixture containing fluorescein-12-dUTP and $\mathrm{TdT}$ for $1 \mathrm{~h}$ at $37^{\circ} \mathrm{C}$. The cell nuclei were stained with 4,6-diamidino-2-phenylindole for $5 \mathrm{~min}$ at room temperature, and the sections were imaged with a fluorescence microscope (Olympus Corporation).

Western blotting. Frozen renal tissues were thawed and total protein extraction was performed by RIPA Lysis and Extraction Buffer (Thermo Fisher Scientific). The proteins were analyzed by immunoblotting using SDS-PAGE (10\%) and transferred to polyvinylidene fluoride membranes. The blots were probed with antibodies against the following proteins: Bcl-2-associated X protein (Bax; 1:1,000, cat. no. 2772), Bcl-2, cleaved-caspase3 (1:1,000, cat. no. 9664), caspase 3 (1:1,000, cat. no. 9662), phosphorylated (p)-JAK2 (1:1,000, cat. no. 3776), JAK2 (1:1,000, cat. no. 3230), p-STAT3 (1:2,000, cat. no. 9145), STAT3 (1:1,000, cat. no. 12640), p-p38MAPK (1:1,000, cat. no. 4511), p-IкB $\alpha(1: 1,000$, cat. no. 5209), IкB $\alpha(1: 1,000$, cat. no. 4812), p-p65 (1:1,000, cat. no. 3031), p65 (1:1,000, cat. no. 8242), and GAPDH (1:1,000, cat. no. 5174; all
Table I. Content of fish oil.

Main components

Contents $(\mathrm{g} / 100 \mathrm{ml})$

Fish oil

10

EPA

$1.25-2.82$

DHA

$1.44-3.09$

Myristic acid

$0.1-0.6$

Palmitic acid

$0.25-1$

Palmitoleic acid

0.3-0.9

Stearic acid

$0.05-0.2$

Oleic acid

$0.6-1.3$

Linoleic acid

$0.1-0.7$

ALA

$\leq 0.2$

SDA

$0.05-0.4$

Eicosanoic acid

$0.05-0.3$

ARA

$0.1-0.4$

Docosanoic acid

$\leq 0.15$

docosapentaenoic acid

$0.15-0.45$

Vitamin E

$0.015-0.0296$

EPA, eicosapentaenoic acid; DHA, docosahexaenoic acid; ALA, $\alpha$-linoleic acid; SDA, stearidonic acid; ARA, arachidonic acid.

Cell Signaling Technology, Inc.). Incubation with the primary antibodies was performed overnight at $4^{\circ} \mathrm{C}$. The following morning, the membranes were washed and incubated with horseradish peroxidase-conjugated secondary anti-rabbit IgG (1:2,000, cat. no. 7074, Cell Signaling Technology, Inc.) for $2 \mathrm{~h}$. The expression levels of each protein were determined using enhanced chemiluminescence reagents (ChemiDoc ${ }^{\mathrm{TM}} \mathrm{XRS}$, Bio-Rad Laboratories, Inc.). The images were collected and quantified using Quantity One Software v4.6.6 (Bio-Rad Laboratories, Inc.).

Statistical analysis. Experiments were performed in triplicate, and data are presented as mean \pm standard deviation. GraphPad Prism 6 (GraphPad Software, Inc.) software was used to analyze the results. Kaplan-Meier analysis was performed to derive survival curves and survival differences was analyzed by log-rank test. Multiple comparisons were assessed by analysis of variance followed by a Bonferroni post-hoc test. $\mathrm{P}<0.05$ was considered statistically significant.

\section{Results}

Effects of FOs in sepsis on the survival of septic rats. To investigate the effects of FOs on the survival rate of septic rats, the survival rate between the CLP group and FOs-treated group was compared. A total of 8 rats did not survive $48 \mathrm{~h}$ of sepsis induction in the CLP group. The survival rate was reduced to $25 \%$ at $48 \mathrm{~h}$ and reached $0 \%$ at $72 \mathrm{~h}$. Following treatment of the animal with dexamethasone, the survival rate was increased to $69 \%$ within $48 \mathrm{~h}$. The survival rate was estimated to $71 \%$ at $48 \mathrm{~h}$ following FOs intervention. The average survival rate of the FOs and dexamethasone groups was significantly higher than that of the CLP group (Fig. 1A). 


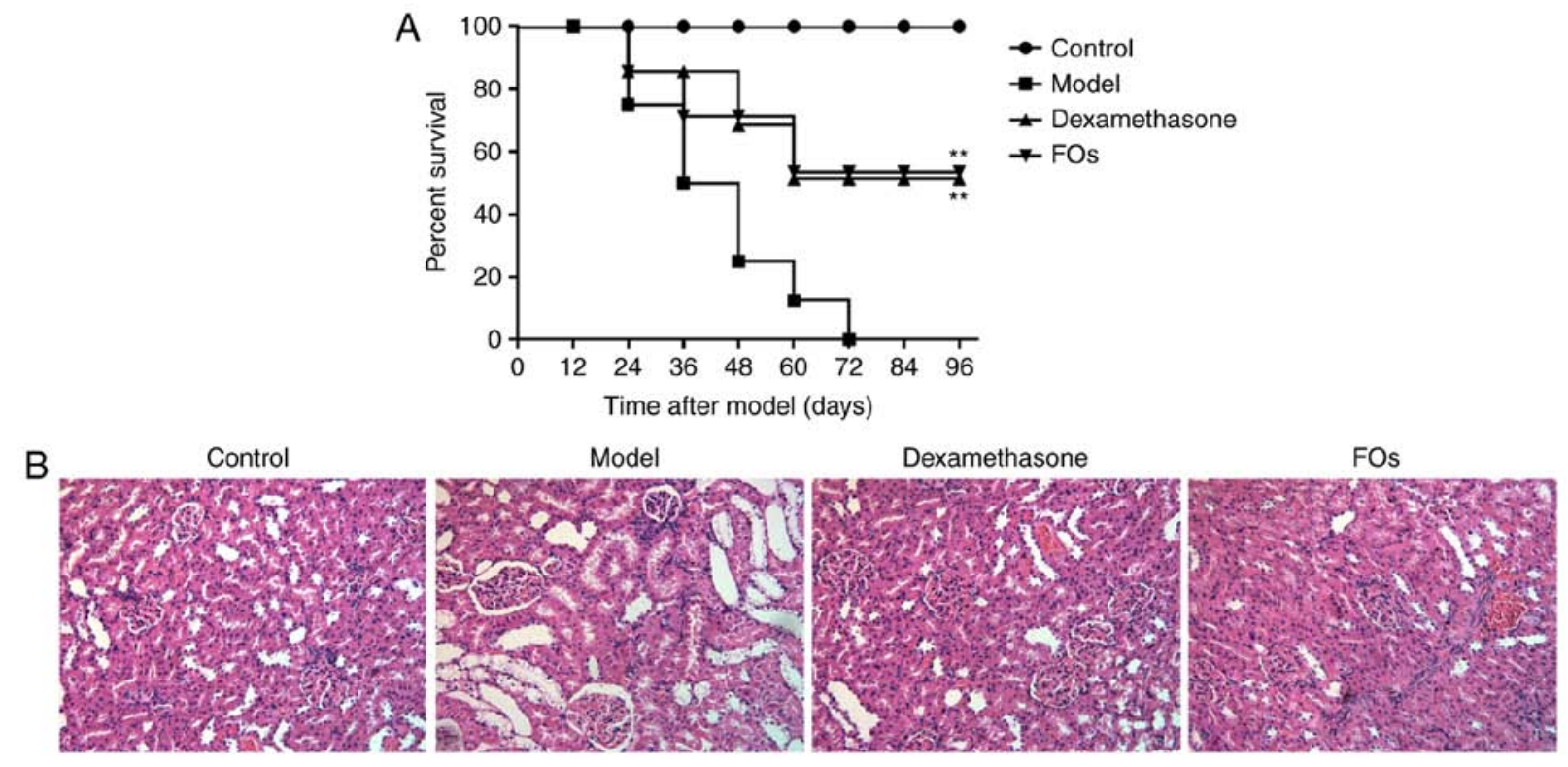

Figure 1. Effects of intraperitoneal administration of FOs on survival and renal tissue microscopic structure of CLP rat. (A) Survival rate of rat in different groups. ${ }^{* *} \mathrm{P}<0.01$ vs. CLP group. (B) Pathological sections of renal tissue were stained with H\&E at $x 100$ magnification. FOs, fish oils; CLP, cecal ligation and puncture.
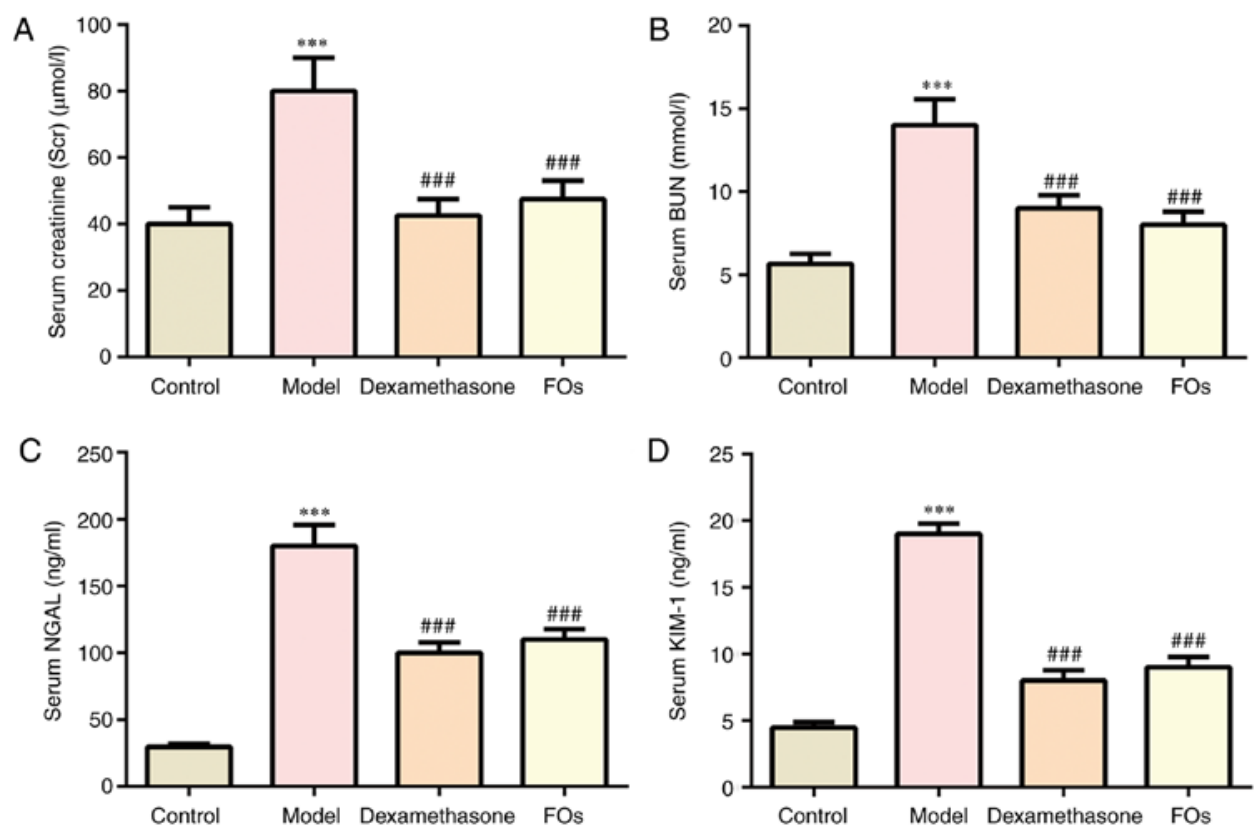

Figure 2. Effects of FOs on CLP-induced renal dysfunction. Levels of (A) Scr, (B) BUN, (C) NGAL and (D) KIM-1 in the four groups. ${ }^{* * *}$ P $<0.001$ vs. Control.

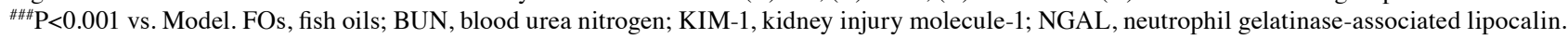

Effects of FOs on renal injury induced by CLP. H\&E staining was performed for histopathological evaluation (Fig. 1B). Histopathological alterations are direct indications of renal injury. In contrast to the control group, the model group presented glomerular atrophy, dilation of the renal capsule cavity, destruction of tubular structures, and local focal epithelial cell necrosis. However, pretreatment with FOs or dexamethasone alleviated glomerular atrophy and epithelial necrosis.

Effects of FOs on CLP-induced renal dysfunction. Renal injury was assessed in rats subjected to CLP for $24 \mathrm{~h}$. The levels of SCr, and BUN, NGAL and KIM-1 in the serum were significantly elevated in the model group at $24 \mathrm{~h}$ post-CLP compared with the control group. However, treatment with dexamethasone and FOs significantly diminished the levels of SCr, BUN, NGAL and KIM-1 compared with those of the model group (Fig. 2).

Effects of FOs on CLP-induced inflammatory cytokines expression in the serum. The expression levels of TNF $\alpha$, IL-1 $\beta$ and IL- 6 were determined by ELISA and by immunofluorescence assays in serum samples from CLP-rats. The results indicated that the expression levels were significantly increased in the model group compared with the control group, whereas administration of FOs or dexamethasone significantly 
A
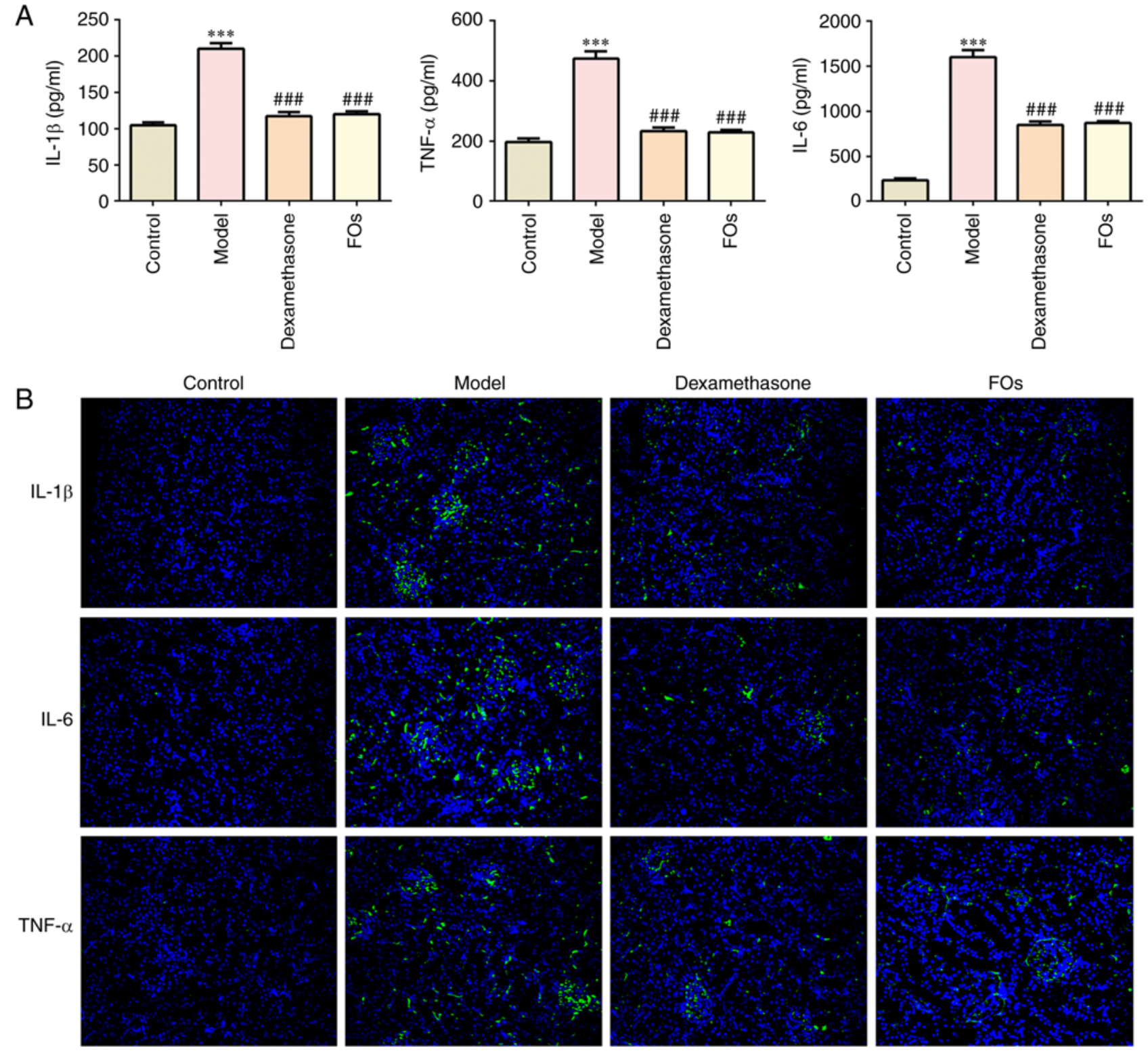

Figure 3. Analysis of inflammatory cytokines. Expression of (A) IL-1 $\beta$, TNF- $\alpha$ and IL-6 levels in the serum among the four groups. ${ }^{* * *} \mathrm{P}<0.001$ vs. Control.

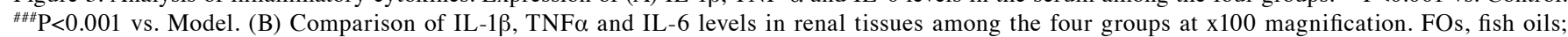
IL, interleukin; TNF $\alpha$, tumor necrosis factor- $\alpha$.

suppressed the induction of TNF $\alpha$, IL-1 $\beta$ and IL- 6 expression in the CLP group (Fig. 3A). Immunofluorescence analysis of the renal tissue samples verified the aforementioned results (Fig. 3B). The data indicated that FOs could reduce the induction of inflammation in the CLP group.

Effects of FOs on CLP-induced oxidative stress and apoptosis. The induction of inflammation is usually accompanied with the induction of oxidative stress and apoptosis (28). The effects of FOs on CLP-induced renal oxidation injury were presented in Fig. 4A. The results indicated that CLP induced a significant increase in the MDA levels compared with the control group. In addition, the activity of the antioxidant enzymes SOD and GSH-Px was significantly decreased in the CLP model compared with the control group. The MDA levels of the CLP rats that received treatment with dexamethasone and FOs were reduced by 60.4 and $57.7 \%$ compared with those of the CLP model group. Moreover, the activity levels of SOD and GSH-Px in animals treated with dexamethasone were increased by 135.8 and $134.5 \%$, whereas FOs treatment resulted in increases of 104.0 and $104.3 \%$, respectively.

TUNEL staining. The induction of apoptosis in the present animal model was stimulated by CLP treatment (Fig. 4B). The percentage of TUNEL-positive cells was increased in the CLP model group compared with the control group. Treatment of the rats with dexamethasone and FOs markedly decreased the number of apoptotic cells. Moreover, the expression levels of the apoptosis-related proteins were detected (Fig. 4C). Bcl-2 expression levels were significantly reduced, whereas the expression levels of Bax and cleaved-caspase 3 were significantly increased in the CLP model compared with those of the control (Fig. 4C). The ratio of Bax/Bcl-2 was significantly increased in the model group, while dexamethasone and 

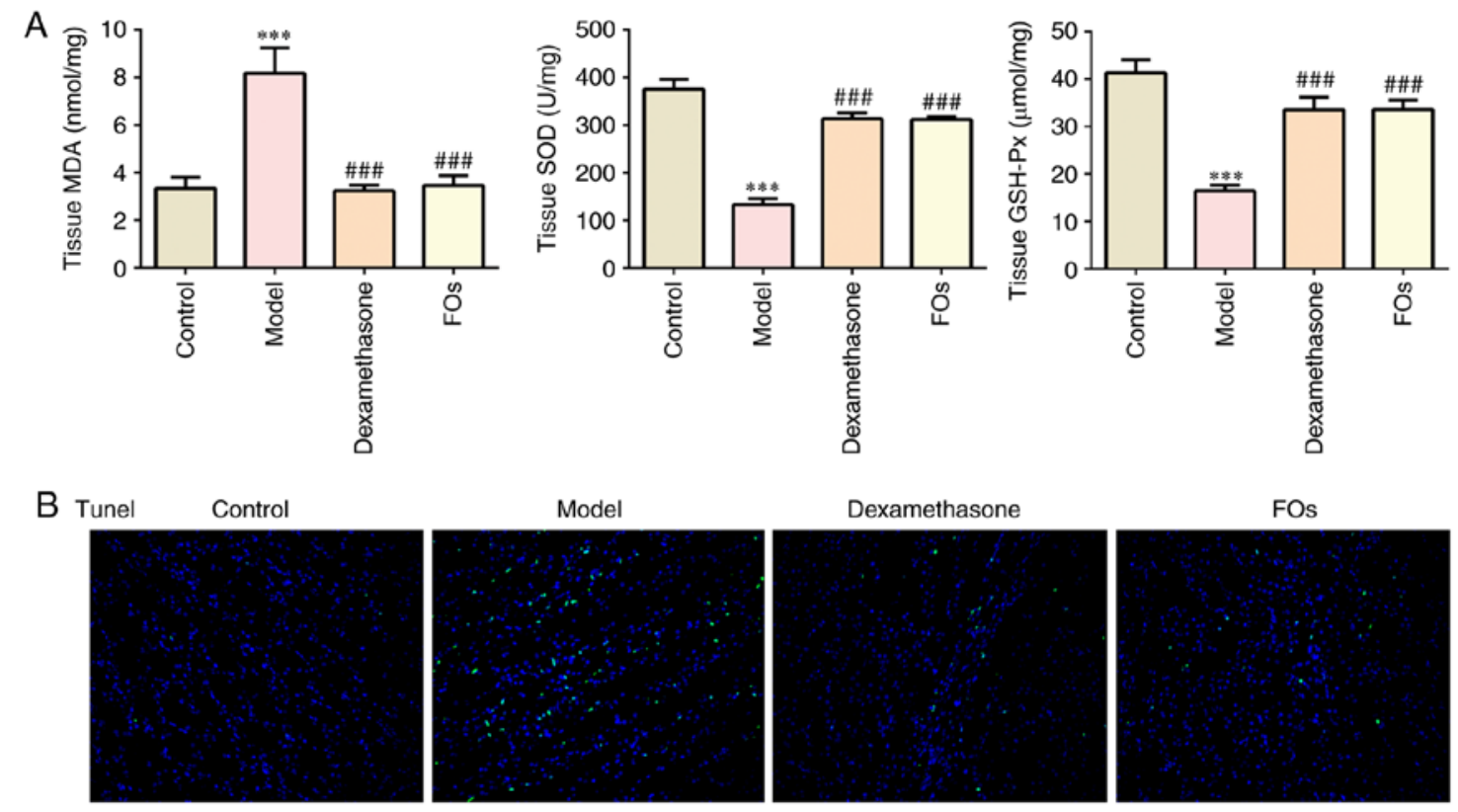

C
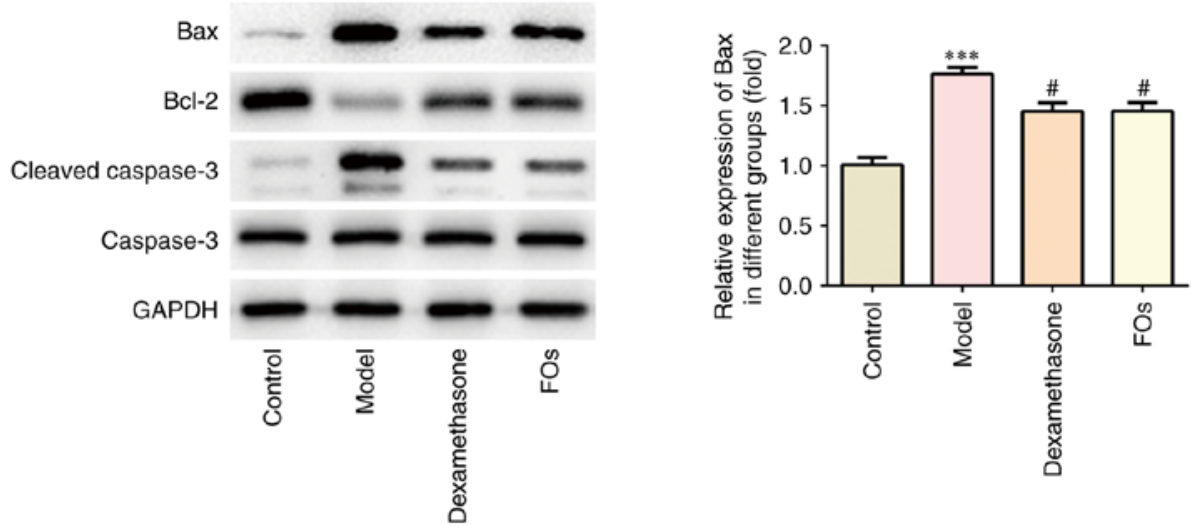
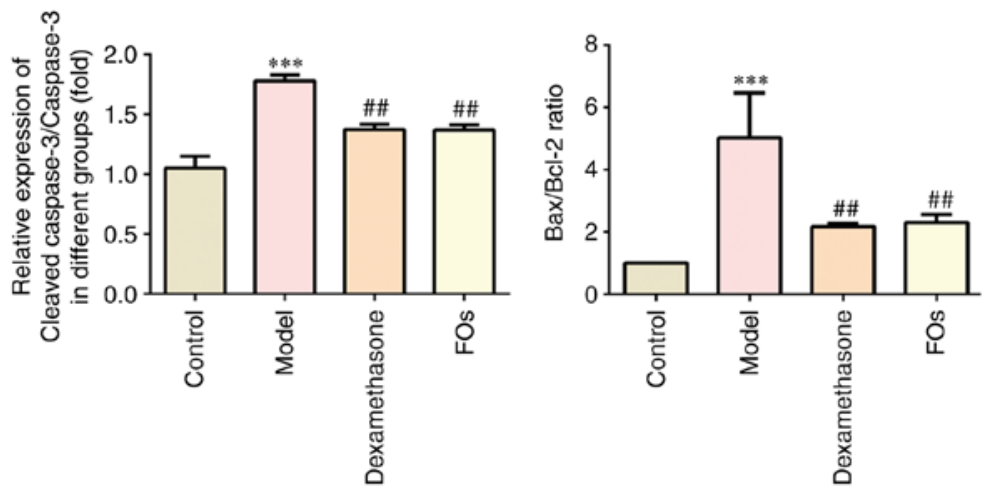

Figure 4. Effects of FOs on oxidative stress and apoptosis. (A) Comparison of MDA, SOD and GSH-Px levels among the four groups. (B) Apoptosis was determined by TUNEL analysis during four groups at x100 magnification. (C) The expression of apoptotic proteins (Bax, Bcl-2, Caspase3) in four groups. ${ }^{* * * *} \mathrm{P}<0.001$ vs. Control. ${ }^{\#} \mathrm{P}<0.05,{ }^{\# \#} \mathrm{P}<0.01,{ }^{\# \#} \mathrm{P}<0.001$ vs. Model. Bax, Bcl-2-associated X protein; FOs, fish oils; GSH-Px, glutathione peroxidase; SOD, superoxide dismutase; TUNEL, Terminal deoxynucleotidyl-transferase-mediated dUTP nick end labelling.

FOs treatment significantly reduced the ratio (Fig. 4C). This indicated the effects of FOs on cell apoptosis. Therefore, administration of dexamethasone and FOs caused a notable decline in the induction of apoptosis by CLP.

FOs negatively regulates the activation of JAK/STAT3, p38-MAPK, and nuclear factor- $\kappa B(N F-k B)$ signaling pathways. FOs were determined to affect the activation of
JAK2/STAT3, p38-MAPK and NF- $\kappa B$ proteins in the renal tissues of CLP rats. Our results indicated that the phosphorylation levels of JAK2, STAT3, p38MAPK, ІкB $\alpha$ and p65 were significantly reduced in renal tissues of CLP rats treated with FOs compared with the model group (Fig. 5).

Histology. H\&E staining of intestinal tissues derived from the CLP model group revealed renal injury as demonstrated by 

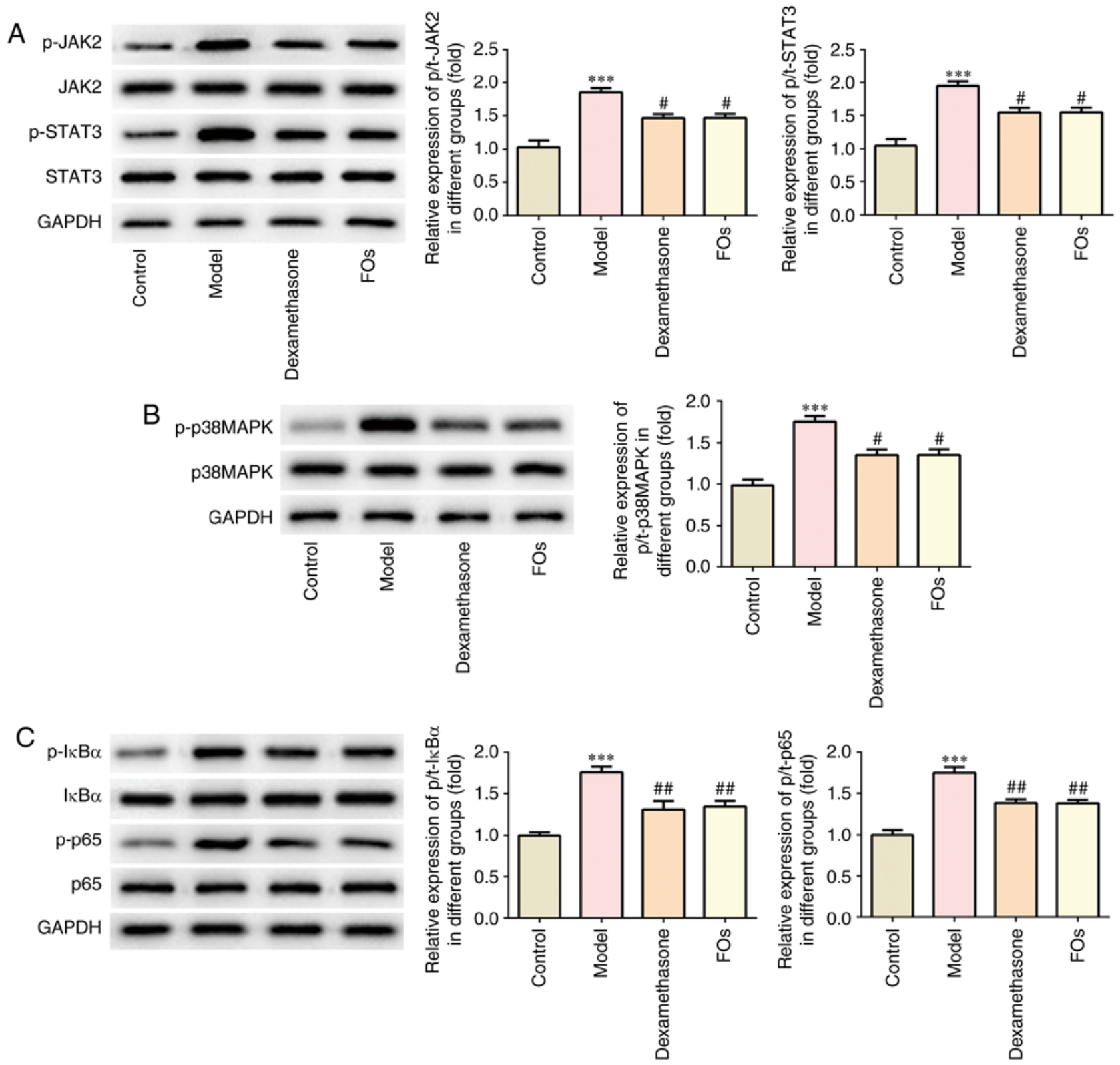

Figure 5. Effects of FOs on JAK2/STAT3, p38-MAPK and NF-кB signaling pathway. (A) Effects of dexamethasone and FOs on JAK2/STAT3 signaling pathway. (B) Effects of dexamethasone and FOs on p38-MAPK signaling pathway. (C) Effects of dexamethasone and FOs on NF- $k B$ signaling pathway.

${ }^{* * * *} \mathrm{P}<0.001$ vs. Control. ${ }^{\#} \mathrm{P}<0.05$ and ${ }^{\# \#} \mathrm{P}<0.01$ vs. Model. FOs, fish oils; $\mathrm{p}$, phosphorylated.

tissue destruction and inflammation. This was reversed by dexamethasone and FOs treatment (Fig. 6A). Immunofluorescence revealed that FO treatment suppressed the production of the pro-inflammatory factors, IL-1 $\beta$, IL- 6 and TNF $\alpha$ in the small intestine of CLP rats (Fig. 6B).

\section{Discussion}

In the present study, the survival rates of rats subjected to CLP-induced sepsis were prolonged following treatment with FOs and dexamethasone. A recent study has shown that in a similar in vivo model, the administration of FOs alone led to $\sim 70 \%$ survival at $48 \mathrm{~h}$ following CLP induction (29). Dexamethasone alleviated the induction of inflammation caused by sepsis and reduced the extent of lipid peroxidation (30). In the present study, dexamethasone was used as a positive control. The current investigation evaluated the therapeutic effects of FOs as a pharmaconutrient in the absence of standard administration of food and water. The levels of SCr, BUN, KIM-1 and NGAL were measured to evaluate kidney injury as described previously (20).

Previous investigations have shown that $\omega 3$ FAs can reduce inflammation, fibrosis and oxidative stress, which are associated with renal function abnormalities (31-33). The protective effects of FOs on AKI and their associated mechanism of action were examined. In the CLP model, kidney injury was evident with distinct changes in the levels of biochemical markers histopathological markers of renal function and injury. The results indicated that treatment of the GLP rats with FOs alleviated the changes in the expression of the histopathological markers, increased the animal survival duration, and decreased the levels of SCr, BUN, KIM-1 and NGAL. 
A

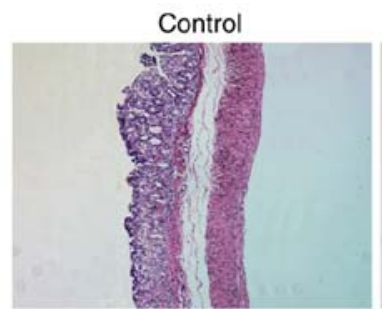

B
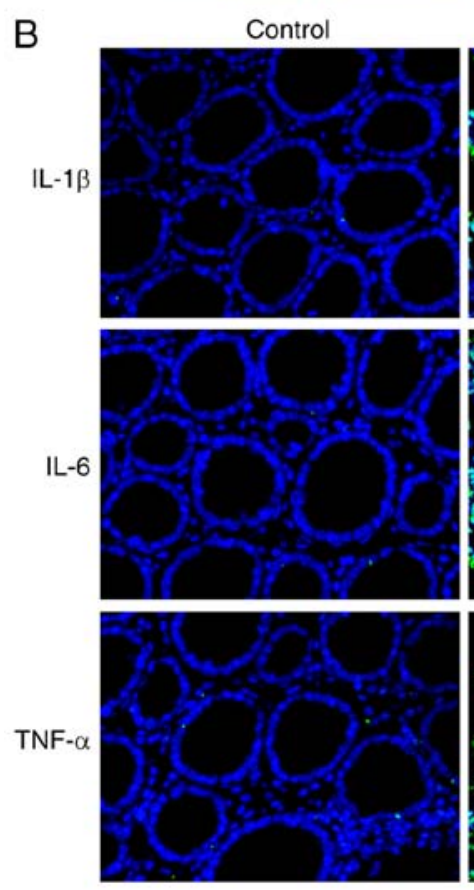

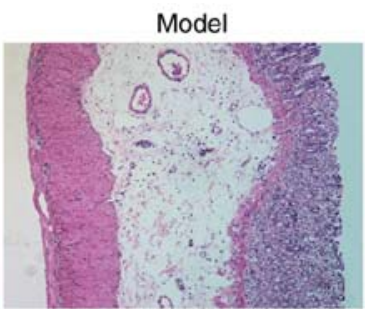

Model
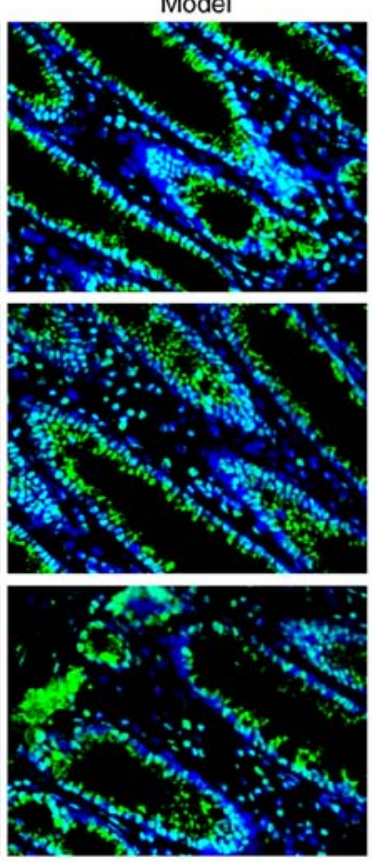

Dexamethasone

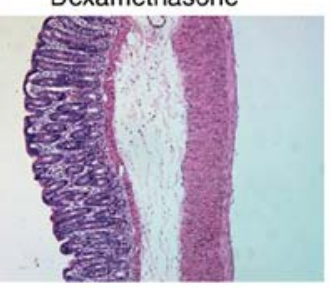

Dexamethasone
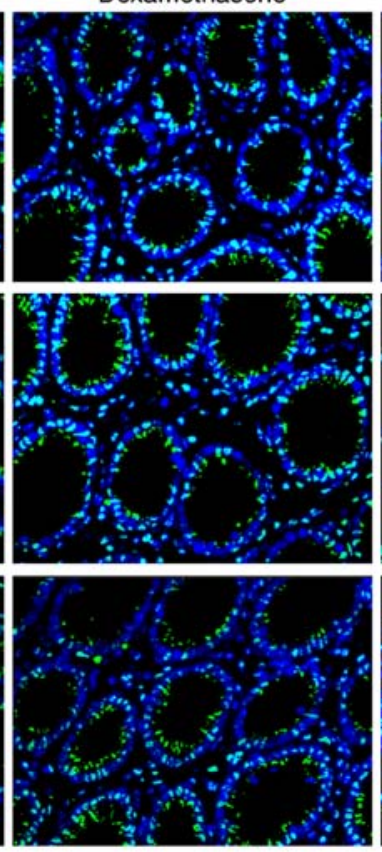

FOs
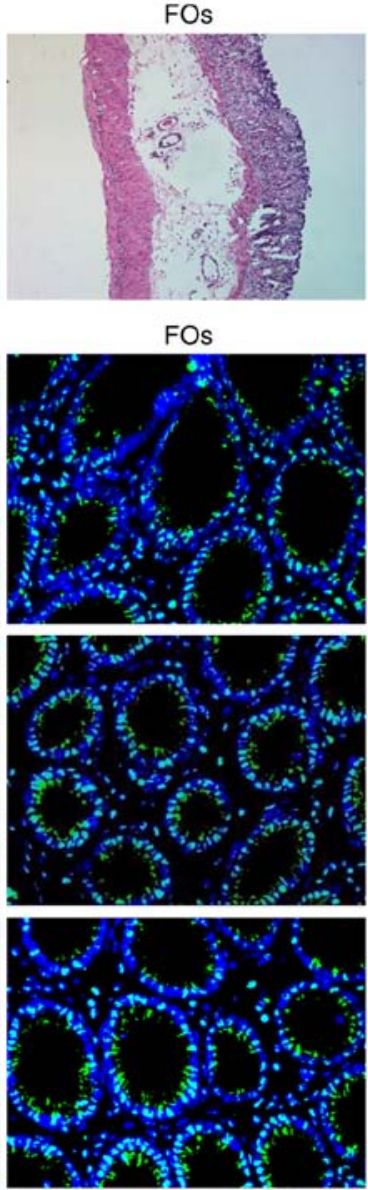

Figure 6. Effects of FOs on septic intestine injury. (A) Pathological sections of intestine were stained with H\&E and examined by microscope at x100 magnification. (B) Comparison of IL-1 $\beta$, TNF $\alpha$ and IL-6 levels in intestinal tissue among the four groups at x100 magnification.

NGAL is a reliable marker for GFR function $(34,35)$. The reduced NGAL levels after pretreatment of FOs suggests that FOs may alleviate renal injury by improving GFR. The downregulation of $\mathrm{SCr}$ and BUN further confirmed this assumption. AKI patients with the existence of KIM-1 expression usually have poor prognosis (36). FOs reduce KIM-1 expression in CLP-induced rats, suggesting the potential effects of FOs on prognosis of sepsis-induced renal injury; however, further investigation is required.

Excessive inflammation is a critical step during septic shock (37). In sepsis, multiple mediators of inflammation participate in organ injury (37). The proinflammatory cytokines IL-1 $\beta$, IL- 6 and TNF $\alpha$ are produced by damaged renal tubule cells or extrarenal cells, serving as potential critical contributors to renal injury (38). The current study confirmed that the anti-inflammatory effects of FOs were mediated by regulation of pro-inflammatory cytokine secretions as reported previously (39). In addition, the data demonstrated that CLP-induced septic intestinal injury could be reduced by FOs treatment. H\&E staining of intestinal tissues derived from the CLP model group confirmed the induction of renal injury as demonstrated by tissue destruction and inflammation. This was reversed by dexamethasone and FOs treatment. In addition, immunofluorescence staining indicated that FOs inhibited the CLP-induced production of the pro-inflammatory factors, IL-1 $\beta$, IL-6 and TNF $\alpha$ in the small intestine. These results supported the aforementioned findings regarding the effects of external FOs on sepsis-induced organ dysfunction. The inhibitory effects of FOs on inflammation may suppress the progression of AKI.

Oxidative stress is a critical step required for the pathogenesis of AKI (40). Oxidative stress, as well as inflammation, can promote the severity of sepsis (41). Excessive oxidative stress is one of the main reason inflammation is induced (41). Early in 1994, FOs have been found to promote antioxidant enzyme expression in murine lupus nephritis (39). Free radicals increased the levels of lipid peroxidation and led to the generation of reactive aldehyde metabolites, such as MDA (42). The antioxidant defense system includes the enzymes (SOD and GSH-Px) that exert protective effects on lipid peroxidation (43). In the present study, FOs caused a significant inhibition in the levels of MDA, and promoted the activity of SOD and GSH-Px in the tissues of septic rats. This suggested that they could suppress CLP-induced oxidative stress by increasing the activity levels of the antioxidant enzymes. Therefore, FOs may attenuate renal injury in the rat CLP model by inhibiting oxidative stress.

Furthermore, the present study reported the suppressive effects of FOs on the induction of apoptosis caused by CLP. The data showed that increased induction of apoptosis was noted in renal tissues from the CLP group, while pretreatment of the rats with FOs could effectively alleviate renal injury by reducing the levels of apoptosis. The expression levels of 
specific apoptotic proteins were determined. The Bcl-2 family of proteins exerts key regulatory functions with regard to apoptosis induction. The Bax to Bcl-2 ratio has been used as a marker for the activation of caspase $3(44,45)$. The present study indicated that the expression levels of cleaved caspase-3 and Bax were increased, whereas the expression levels of Bcl-2 were decreased in the CLP model group. Moreover, pretreatment of the rats with FOs could reverse the changes noted in the expression levels of these proteins. The results indicated that FOs exhibited a strong protective effect against CLP induced apoptosis.

It has been reported that FOs inhibit the $N F-\kappa B$ signaling pathway in sepsis-induced liver injury (46). The activation of p38 has been shown to increase the expression levels of the inflammatory cytokines, such as TNF- $\alpha$, IL- $1 \beta$ and IL- 6 in microglial cells (47). Furthermore, TNF- $\alpha$ serves a critical role in the endothelial dysfunction of patients with sepsis via activating the $\mathrm{p} 38-\mathrm{MAPK}$ and the NF- $\mathrm{B}$ pathways (48). The suppression of the $\mathrm{TNF} / \mathrm{p} 38-\mathrm{MAPK} /$ caspase-3 signaling pathway is also considered a key mechanism in the prevention of sepsis-induced myocardial injury in rats (49). In the present study, the results indicated that FOs inhibited p38-MAPK and NF- $\mathrm{B}$ signaling activation by CLP. In addition, the JAK/STAT signaling pathway is involved in immune and inflammatory responses, by regulating the expression of several target genes $(50,51)$. JAK is an upstream regulator of STAT activation, which has been rarely investigated in sepsis-induced organ dysfunction. In the current study, the activation of JAK2/STAT3 signaling pathway was inhibited by FOs. This was noted in the renal tissues from CLP rats. The results indicated that FOs could inhibit the CLP-induced activation of the NF- $\mathrm{B}, \mathrm{p} 38-\mathrm{MAPK}$, and JAK2/STAT3 signaling pathways. Whether the effects of FOs on renal function and induction of oxidative stress, apoptosis and inflammation were mediated by these signaling pathways remains unclear. Thus, further investigation is required.

\section{Acknowledgements}

Not applicable.

\section{Funding}

No funding was received.

\section{Availability of data and materials}

The datasets generated and/or analyzed during the current study are not publicly available due to data confidentiality in our hospital but are available from the corresponding author on reasonable request.

\section{Authors' contributions}

ZL drafted the manuscript, data analysis, and made substantial contributions to the design of the present study and resolved the problems during investigation. JJ prepared experimental samples and detection. XS prepared the manuscript, and was involved in western blotting and ELISA. All authors read and approved the final manuscript.

\section{Ethics approval and consent to participate}

The animal experiment protocol was approved by the Commission for Animal Experimentation of the people's hospital of Xishuangbanna Dai Nationality Autonomous Prefecture.

\section{Patient consent for publication}

Not applicable.

\section{Competing interests}

The authors declare that they have no competing interests.

\section{References}

1. Singer M, Deutschman CS, Seymour CW, Shankar-Hari M, Annane D, Bauer M, Bellomo R, Bernard GR, Chiche JD, Coopersmith CM, et al: The third international consensus definitions for sepsis and septic shock (Sepsis-3). JAMA 315: 801-810, 2016.

2. Greenberg JH, Coca S and Parikh CR: Long-term risk of chronic kidney disease and mortality in children after acute kidney injury: A systematic review. BMC Nephrol 15: 184, 2014.

3. Honore PM, Jacobs R, Hendrickx I, Bagshaw SM, JoannesBoyau O, Boer W, De Waele E, Van Gorp V and Spapen HD: Prevention and treatment of sepsis-induced acute kidney injury: An update. Ann Intensive Care 5: 51, 2015.

4. Redivo DDB, Jesus CHA, Sotomaior BB, Gasparin AT and Cunha JM: Acute antinociceptive effect of fish oil or its major compounds, eicosapentaenoic and docosahexaenoic acids on diabetic neuropathic pain depends on opioid system activation. Behav Brain Res 372: 111992, 2019.

5. Cao S, Ren J, Sun L, Gu G, Yuan Y and Li J: Fish oil-supplemented parenteral nutrition prolongs survival while beneficially altering phospholipids' fatty acid composition and modulating immune function in rat sepsis. Shock 36: 184-190, 2011.

6. Ostermann M and Chang RW: Acute kidney injury in the intensive care unit according to RIFLE. Crit Care Med 35: 1837-1843; quiz 1852, 2007.

7. Tancevski I, Nairz M, Duwensee K, Auer K, Schroll A, Heim C, Feistritzer C, Hoefer J, Gerner RR, Moschen A, et al: Fibrates ameliorate the course of bacterial sepsis by promoting neutrophil recruitment via CXCR2. EMBO Mol Med 6: 810-820, 2014.

8. Napier BA, Andres-Terre M, Massis LM, Hryckowian AJ, Higginbottom SK, Cumnock K, Casey KM, Haileselassie B, Lugo KA, Schneider DS, et al: Western diet regulates immune status and the response to LPS-driven sepsis independent of diet-associated microbiome. Proc Natl Acad Sci USA 116: 3688-3694, 2019.

9. Cao YZ, Tu YY, Chen X, Wang BL, Zhong YX and Liu MH: Protective effect of Ulinastatin against murine models of sepsis: Inhibition of TNF- $\alpha$ and IL- 6 and augmentation of IL-10 and IL-13. Exp Toxicol Pathol 64: 543-547, 2012.

10. Hotchkiss RS, Monneret G and Payen D: Sepsis-induced immunosuppression: From cellular dysfunctions to immunotherapy. Nat Rev Immunol 13: 862-874, 2013.

11. Saglimbene VM, Wong G, van Zwieten A, Palmer SC, Ruospo M, Natale P, Campbell K, Teixeira-Pinto A, Craig JC and Strippoli GFM: Effects of omega-3 polyunsaturated fatty acid intake in patients with chronic kidney disease: Systematic review and meta-analysis of randomized controlled trials. Clin Nutr, Mar 14, 2019 (Epub ahead of print).

12. Zhou Q, Zhang Z, Wang P, Zhang B, Chen C, Zhang C and Su Y: EPA+DHA, but not ALA, improved lipids and inflammation status in hypercholesterolemic adults: A randomized, double-blind, placebo-controlled trial. Mol Nutr Food Res 63: e1801157, 2019.

13. Shih JM, Shih YM, Pai MH, Hou YC, Yeh CL and Yeh SL: Fish oil-based fat emulsion reduces acute kidney injury and inflammatory response in antibiotic-treated polymicrobial septic mice. Nutrients 8: 165, 2016. 
14. Quoilin C, Mouithys-Mickalad A, Lecart S, Fontaine-Aupart MP and Hoebeke M: Evidence of oxidative stress and mitochondrial respiratory chain dysfunction in an in vitro model of sepsis-induced kidney injury. Biochim Biophys Acta 1837: 1790-1800, 2014.

15. Abd-Ellatif RN, Hegab II, Atef MM, Sadek MT and Hafez YM: Diacerein protects against glycerol-induced acute kidney injury: Modulating oxidative stress, inflammation, apoptosis and necroptosis. Chem Biol Interact 306: 47-53, 2019.

16. Heller AR, Rössler S, Litz RJ, Stehr SN, Heller SC, Koch R and Koch T: Omega-3 fatty acids improve the diagnosis-related clinical outcome. Crit Care Med 34: 972-979, 2006.

17. Leelahavanichkul A, Souza AC, Street JM, Hsu V, Tsuji T, Doi K, Li L, Hu X, Zhou H, Kumar P, et al: Comparison of serum creatinine and serum cystatin $\mathrm{C}$ as biomarkers to detect sepsis-induced acute kidney injury and to predict mortality in CD-1 mice. Am J Physiol Renal Physiol 307: F939-F948, 2014.

18. Nair S, O'Brien SV, Hayden K, Pandya B, Lisboa PJ, Hardy KJ and Wilding JP: Effect of a cooked meat meal on serum creatinine and estimated glomerular filtration rate in diabetes-related kidney disease. Diabetes Care 37: 483-487, 2014.

19. Blantz RC: Pathophysiology of pre-renal azotemia. Kidney Int 53: 512-523, 1998.

20. Luo QH, Chen ML, Chen ZL, Huang C, Cheng AC, Fang J, Tang L and Geng Y: Evaluation of KIM-1 and NGAL as early indicators for assessment of gentamycin-induced nephrotoxicity in vivo and in vitro. Kidney Blood Press Res 41: 911-918, 2016.

21. Rysz J, Gluba-Brzózka A, Franczyk B, Jabłonowski Z and Ciałkowska-Rysz A: Novel biomarkers in the diagnosis of chronic kidney disease and the prediction of its outcome. Int J Mol Sci 18: pii: E1702, 2017.

22. Yuan SM: Acute kidney injury after cardiac surgery: Risk factors and novel biomarkers. Braz J Cardiovasc Surg 34: 352-360, 2019.

23. Doi K, Leelahavanichkul A, Yuen PS and Star RA: Animal models of sepsis and sepsis-induced kidney injury. J Clin Invest 119: 2868-2878, 2009.

24. Rittirsch D, Huber-Lang MS, Flierl MA and Ward PA: Immunodesign of experimental sepsis by cecal ligation and puncture. Nat Protoc 4: 31-36, 2009.

25. Stevens NE, Chapman MJ, Fraser CK, Kuchel TR, Hayball JD and Diener KR: Therapeutic targeting of HMGB1 during experimental sepsis modulates the inflammatory cytokine profile to one associated with improved clinical outcomes. Sci Rep 7: 5850, 2017.

26. Meng L, Li L, Lu S, Li K, Su Z, Wang Y, Fan X, Li X and Zhao G: The protective effect of dexmedetomidine on LPS-induced acute lung injury through the HMGB1-mediated TLR4/NF- $\mathrm{KB}$ and PI3K/Akt/mTOR pathways. Mol Immunol 94: 7-17, 2018.

27. Jia Y, Li Z, Feng Y, Cui R, Dong Y, Zhang X, Xiang X, Qu K, Liu C and Zhang J: Methane-rich saline ameliorates sepsis-induced acute kidney injury through anti-inflammation, antioxidative, and antiapoptosis effects by regulating endoplasmic reticulum stress. Oxid Med Cell Longev 2018: 4756846, 2018.

28. Hussain T, Tan B, Yin Y, Blachier F, Tossou MC and Rahu N: Oxidative stress and inflammation: What polyphenols can do for us? Oxid Med Cell Longev 2016: 7432797, 2016.

29. Tian T, Zhao Y, Huang Q and Li J: N-3 polyunsaturated fatty acids improve inflammation via inhibiting sphingosine kinase 1 in a rat model of parenteral nutrition and CLP-induced sepsis. Lipids 51: 271-278, 2016.

30. Shalmani AA, Ghahremani MH, Jeivad F, Shadboorestan A, Hassanzadeh G, Beh-Pajooh A, Ganbari-Erdi M, Kasirzadeh S Mojtahedzadeh M and Sabzevari O: Monomethyl fumarate alleviates sepsis-induced hepatic dysfunction by regulating TLR-4/NF- $\kappa$ B signalling pathway. Life Sci 215: 152-158, 2018.

31. Peake JM, Gobe GC, Fassett RG and Coombes JS: The effects of dietary fish oil on inflammation, fibrosis and oxidative stress associated with obstructive renal injury in rats. Mol Nutr Food Res 55: 400-410, 2011.

32. An WS, Kim HJ, Cho KH and Vaziri ND: Omega-3 fatty acid supplementation attenuates oxidative stress, inflammation, and tubulointerstitial fibrosis in the remnant kidney. Am J Physiol Renal Physiol 297: F895-F903, 2009.

33. Jangale NM, Devarshi PP, Bansode SB, Kulkarni MJ and Harsulkar AM: Dietary flaxseed oil and fish oil ameliorates renal oxidative stress, protein glycation, and inflammation in streptozotocin-nicotinamide-induced diabetic rats. J Physiol Biochem 72: 327-336, 2016.

34. Bolignano D, Donato V, Coppolino G, Campo S, Buemi A, Lacquaniti A and Buemi M: Neutrophil gelatinase-associated lipocalin (NGAL) as a marker of kidney damage. Am J Kidney Dis 52: 595-605, 2008.
35. McIlroy DR, Wagener G and Lee HT: Neutrophil gelatinaseassociated lipocalin and acute kidney injury after cardiac surgery: The effect of baseline renal function on diagnostic performance. Clin J Am Soc Nephrol 5: 211-219, 2010.

36. Tu Y, Wang H, Sun R, Ni Y, Ma L, Xv F, Hu X, Jiang L, Wu A, Chen X, et al: Urinary netrin-1 and KIM-1 as early biomarkers for septic acute kidney injury. Ren Fail 36: 1559-1563, 2014.

37. You B, Zhang YL, Luo GX, Dang YM, Jiang B, Huang GT, Liu XZ, Yang ZC, Chen Y, Chen J, et al: Early application of continuous high-volume haemofiltration can reduce sepsis and improve the prognosis of patients with severe burns. Crit Care 22: 173,2018

38. Park SW, Chen SW, Kim M, Brown KM, Kolls JK, D'Agati VD and Lee HT: Cytokines induce small intestine and liver injury after renal ischemia or nephrectomy. Lab Invest 91: 63-84, 2011.

39. Chandrasekar B and Fernandes G: Decreased pro-inflammatory cytokines and increased antioxidant enzyme gene expression by omega-3 lipids in murine lupus nephritis. Biochem Biophys Res Commun 200: 893-898, 1994.

40. Pavlakou P, Liakopoulos V, Eleftheriadis T, Mitsis M and Dounousi E: Oxidative stress and acute kidney injury in critical illness: Pathophysiologic mechanisms-biomarkers-interventions, and future perspectives. Oxid Med Cell Longev 2017: 6193694, 2017.

41. Galley HF: Oxidative stress and mitochondrial dysfunction in sepsis. Br J Anaesth 107: 57-64, 2011.

42. Levent G, Ali A, Ahmet A, Polat EC, Aytaç C, Ayşe E and Ahmet S: Oxidative stress and antioxidant defense in patients with chronic hepatitis $\mathrm{C}$ patients before and after pegylated interferon alfa-2b plus ribavirin therapy. J Transl Med 4: 25, 2006.

43. Segura-Aguilar J, Cortes-Vizcaino V, Llombart-Bosch A, Ernster L, Monsalve E and Romero FJ: The levels of quinone reductases, superoxide dismutase and glutathione-related enzymatic activities in diethylstilbestrol-induced carcinogenesis in the kidney of male Syrian golden hamsters. Carcinogenesis 11: 1727-1732, 1990.

44. McClintock DS, Santore MT, Lee VY, Brunelle J, Budinger GR, Zong WX, Thompson CB, Hay N and Chandel NS: Bcl-2 family members and functional electron transport chain regulate oxygen deprivation-induced cell death. Mol Cell Biol 22: 94-104, 2002.

45. Dang J, Jia R, Tu Y, Xiao S and Ding G: Erythropoietin prevents reactive oxygen species generation and renal tubular cell apoptosis at high glucose level. Biomed Pharmacother 64: 681-685, 2010.

46. Li CC, Yang HT, Hou YC, Chiu YS and Chiu WC: Dietary fish oil reduces systemic inflammation and ameliorates sepsis-induced liver injury by up-regulating the peroxisome proliferator-activated receptor gamma-mediated pathway in septic mice. J Nutr Biochem 25: 19-25, 2014.

47. Taves S, Berta T, Liu DL, Gan S, Chen G, Kim YH, Van de Ven T, Laufer S and Ji RR: Spinal inhibition of p38 MAP kinase reduces inflammatory and neuropathic pain in male but not female mice: Sex-dependent microglial signaling in the spinal cord. Brain Behav Immun 55: 70-81, 2016.

48. Liang Y, Li X, Zhang X, Li Z, Wang L, Sun Y, Liu Z and Ma X: Elevated levels of plasma TNF- $\alpha$ are associated with microvascular endothelial dysfunction in patients with sepsis through activating the NF- $\kappa \mathrm{B}$ and $\mathrm{p} 38$ mitogen-activated protein kinase in endothelial cells. Shock 41: 275-281, 2014.

49. Zhang M, Wang X, Bai B, Zhang R, Li Y and Wang Y: Oxymatrine protects against sepsis-induced myocardial injury via inhibition of the TNF- $\alpha /$ p38-MAPK/caspase-3 signaling pathway. Mol Med Rep 14: 551-559, 2016.

50. Pena G, Cai B, Liu J, van der Zanden EP, Deitch EA, de Jonge WJ and Ulloa L: Unphosphorylated STAT3 modulates alpha 7 nicotinic receptor signaling and cytokine production in sepsis. Eur J Immunol 40: 2580-2589, 2010.

51. Yuan FH, Chen YL, Zhao Y, Liu ZM, Nan CC, Zheng BL, Liu XY and Chen XY: microRNA-30a inhibits the liver cell proliferation and promotes cell apoptosis through the JAK/STAT signaling pathway by targeting SOCS-1 in rats with sepsis. J Cell Physiol 234: 17839-17853, 2019.

This work is licensed under a Creative Commons Attribution-NonCommercial-NoDerivatives 4.0 International (CC BY-NC-ND 4.0) License. 